\title{
Performance of The ATLAS Muon Spectrometer During the Commissioning
}

\author{
Claudio Ferretti ${ }^{* \dagger}$ \\ University of Michigan \\ E-mail: Claudiof@umich.edu
}

The ATLAS detector has been operating for several months: in the fall 2008 the first proton beam circulated in the LHC machine but no beam-beam collisions have been produced yet, so that the data collected so far are mainly cosmic rays. The very large ATLAS Muon Spectrometer (MS) includes four different technology chamber types. It has been designed to trigger on single muons of pseudo-rapidity up to 2.4 and transverse momentum down to a few $\mathrm{GeV}$ and track reconstruction with a standalone momentum resolution better than $10 \%$ for particles up to a few $\mathrm{TeV}$. The Muon system, while still in the process of being completed, has undergone an intense program of commissioning, ranging from understanding the status of each single chamber to study the performance of the whole system for triggering, data acquisition, and reconstruction software. Several preliminary results relevant to the muon trigger and reconstruction performance are here presented.

European Physical Society Europhysics Conference on High Energy Physics, EPS-HEP 2009, July 16 - 222009

Krakow, Poland

\footnotetext{
*Speaker.
} 


\section{Introduction: The ATLAS Muon Spectrometer}

Three superconducting air-core toroids (one in the barrel and one for each end-cap), with a typical field integral in the range between 2 and $8 \mathrm{Tm}$ provide the magnetic field. The muon momentum is measured from its curvature reconstructed in the MS by using three stations of precision chambers positioned inside a toroidal magnetic field along its trajectory.

The precision chambers are Monitored Drift Tubes (MDT) and for large pseudorapidities $(|\eta|>2$, where the background rate is higher) by the Cathode Strip Chambers (CSC). For a 1 $\mathrm{TeV}$ muon track the sagitta (residual in the middle station repect to a straight track due to its curvature) varies between $0.5 \mathrm{~mm}$ at $\eta=0$ and $1 \mathrm{~mm}$ at $\eta=2$. A momentum resolution of $10 \%$ requires that the error on the sagitta measurement less than $50 \mu \mathrm{m}$ in the bending direction of the B-field, transverse to the MDT tubes and wires. The intrinsic resolution of the MDTs results in a sagitta error of $\sim 40 \mu \mathrm{m}$, and the additional error from the chamber alignment (3D-position, rotations and deformations) should not exceed that value.

The trigger is provided by Resistive Plate Chambers (RPC) in the barrel region $(|\eta|<1)$ while the Thin Gap Chambers (TGC) are used in the higher background environment of the end-cap region $(|\eta|<2.4)$. RPC and TGC provide also the "second" (non-bending) coordinate along the MDT tubes that cannot be measured by the precision chambers.

A more detailed description of the ATLAS muon spectrometer and of its expected performance

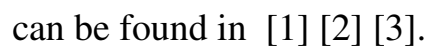

\section{Hardware Status of the Muon Spectrometer}

Most of the cosmic rays reach the ATLAS experiment via the two big shafts. They have incident angles very close to the vertical axis and they are mainly triggered by the Barrel trigger chambers (RPC). All 606 RPC and 3588 TGC chambers are installed in the ATLAS cavern for a total of respectively 373,000 and 318,000 channels and the current fractions of working chambers is $\sim 95.5 \%$ (RPC) and over 99.9\% (TGC). Several hundred million cosmic ray events were acquired during 2008 and more in the current year in preparation for collisions from the LHC mostly using muon trigger, showing that the MS trigger system is working reliably, with the tuning of the timing between stations is in progress (and completed for the TGC) and between sub-detector will need collision data. For the precision chambers, all 32 CSC and 1090/1150 MDT are installed (where part the initially delayed 'EE' MDT's are currently being mounted on the detector) for a total of 30,700 (CSC) and 354,000 (MDT) channels. Over $99.5 \%$ of the MDT channels and $98.5 \%$ of the CSC layers are operational.

Even just limiting to the MDT sub-system, there are over 18 thousand fromt end electronic cards to be monitored (voltages and temperatures), almost 2,000 Hall probes to measures the local magnetic filed intensity and direction, over 13,000 temperature sensors, etc and for each one of them the fraction of those not working is well below $1 \%$. Furthermore, to monitor the chamber alignment is used a grid of $\approx 12,000$ optical sensors: currently over $99 \%$ are working and the redundancy makes negligible the degradation due to as few missing sensors. 


\section{Muon Spectrometer Performance}

The muon track is reconstructed by fitting the segments built in single precision chambers, being a segment just a linear fit to the hits. In order to use the MDT hits one has to know the global time offset $\left(t_{0}\right.$, affected on the current data by a $25 \mathrm{~ns}$ jitter due to the asynchronicity of the cosmic rays with the LHC clock) and the drift time-space relationship (RT function, not linear and depending on B-field, temperature, gas pressure, etc). The calibration procedure is now able to prepare this quantities for the reconstruction for each run in 24-36 hours.

The single hit resolution, measured by refitting a good segment (with hits on all layers) where one hit at the time was removed and measuring the residuals of the excluded hit versus the radius, is in excellent agreement with the one measured during the test beam once added a couple of ns due to trigger timing correction (see Fig. 11 left).

In case ideal case of perfect alignment and with magnetic field off, the sagitta of a muon track is expected to be dominated by the multiple scattering and has mean zero: in Fig. 11-middle the mean of the sagitta is $15 \pm 42 \mu \mathrm{m}$. A track based alignment (especially for barrel small sectors) used in conjuction with the optical ones has given an average sagitta $22 \pm 7 \mu \mathrm{m}$ for a "tuned" chamber.

A cosmic muon passing trough the detector can be reconstructed in the spectrometer as two tracks split at the perigee (closest approach to the beam axis). The comparison of the upper and lower tracks momentum at the external surface of the calorimiter shows an average energy loss of $6 \mathrm{GeV}$. The MS tracks is then extrapolated to the perigee taking into account both the scattering and the energy loss inside the calorimeter and compared to the same track reconstructed by the inner detector (ID). This way one can learn about the absolute energy scale and the misalignment between MS and ID. In Fig. 11 right the curvatures found by the two sub-detectors are very similar, showing that the misalignment are limited and the reconstruction produces very similar results.
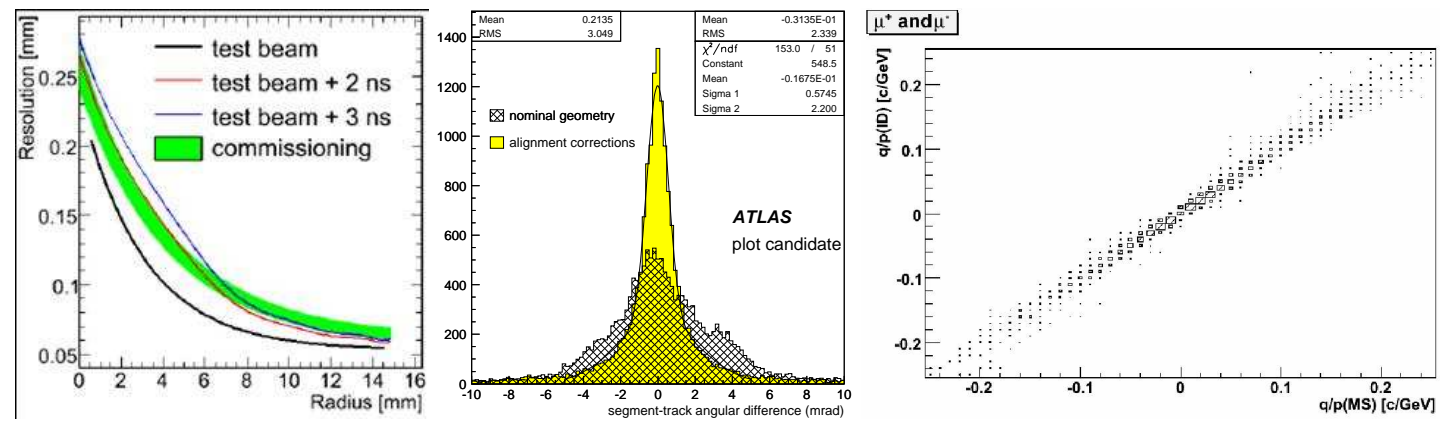

Figure 1: (left) MDT single hit resolution in test beam and 2008 data as function of the tube radius R. (middle) MDT sagitta for runs without B-field before (hatched) and after (yellow) alignment corrections. (right) Muon Spectrometer $q / P(\mathrm{X}$ axis) vs Inner Detector $q / P(\mathrm{Y}$ axis)

\section{References}

[1] The ATLAS Muon Spectrometer Technical Design Report, The ATLAS Muon Collaboration, CERN/LHCC/97-22, 31 May 1997.

[2] The ATLAS Experiment at the CERN Large Hadron Collider, The ATLAS Collaboration, G Aad et al 2008 JINST 3 S08003.

[3] Expected Performance of the ATLAS Experiment: Detector, Trigger and Physics The ATLAS Collaboration, G Aad et al., CERN-OPEN 2008-020 